\title{
Robust Predictive Quantization: A New Analysis and Optimization Framework
}

\author{
Alyson K. Fletcher, ${ }^{1}$ Sundeep Rangan, ${ }^{2}$ Vivek K Goyal, ${ }^{3}$ and Kannan Ramchandran ${ }^{1}$ \\ ${ }^{1}$ Dept. of EECS, University of California, Berkeley, Cory Hall, Berkeley, CA 94720-1770 USA \\ ${ }^{2}$ Flarion Technologies, Bedminster One, 135 Route 202/206 South, Bedminster, NJ 07921 USA \\ ${ }^{3}$ Dept. of EECS, Massachusetts Institute of Technology, 77 Massachusetts Ave., Cambridge, MA 02139 USA \\ e-mail: alyson@eecs.berkeley.edu, s.rangan@flarion.com, vgoyal@mit.edu, kannanr@eecs.berkeley.edu
}

\begin{abstract}
This work is focused on computingvia a deterministic optimization with linear matrix inequality (LMI) constraints, rather than a pseudorandom simulation - the performance of predictive quantization schemes under various scenarios for loss and degradation of encoded prediction error samples. The ability to make this computation then allows for the optimization of prediction filters with the aim of minimizing overall mean squared error (including the effects of losses) rather than to minimize the variance of the unquantized prediction error sequence. The main tools are recent characterizations of asymptotic state estimation error covariance and output estimation error variance in terms of LMIs. These characterizations apply to discrete-time jump linear systems in which the discrete portion of the system state is a Markov chain. Translating to the signal processing terminology, this means that the signal model is "piecewise ARMA," as is standard in many forms of speech processing.
\end{abstract}

Prediction is fundamental to source coding, and in particular, predictive quantization is one of the simplest ways to exploit the memory of a random process in lossy source coding. Predictive quantization is used in almost all practical speech coding, and in the slightly more complicated form of motion compensation, in most current video coding. However, prediction is problematic when there are uncorrectable losses between the encoder and the decoder; the decoder is forced to "predict" based on data it simply does not have.

With existing tools, it is difficult is analyze the overall performance of predictive quantization with losses, i.e., to quantify the effect on mean-squared error (MSE) of the losses. Thus, it is difficult to assess whether the prediction is desirable, let alone to improve upon the standard encoding. Our aim is to provide a methodology for optimizing the "prediction filter" for overall performance. The solution may not be an optimal prediction filter in the conventional sense because it does not minimize the variance of the prediction error sequence; rather, the optimizing filter is the key component of a type of joint source-channel code.

We apply a framework introduced in [1] to the system shown in Fig. 1 by modeling the signal to be compressed is an output of a jump linear system driven by a white noise input. Losses of encoded prediction errors can be modeled by any Markov chain, and in fact any linear degradation can also be included. Thus, this approach has considerable generality. Two weaknesses should be noted, however: a) we must adopt the technically incorrect (but common and useful) stochastic model for quantization error whereby the error is independent of the input; and b) we assume a linear reconstruction at the

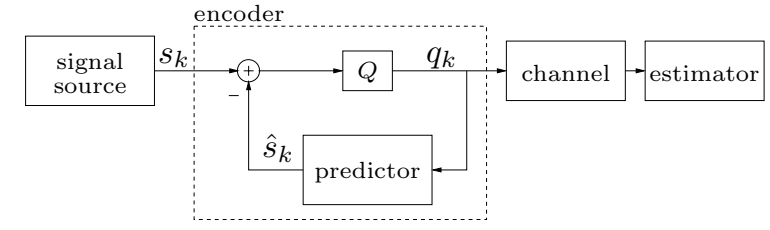

Figure 1: Abstract model of a system with a predictive quantizer. The analysis replaces the signal source with a state-space system $H_{s}=\left(A_{s}, B_{s}, C_{s}, D_{s}\right)$, the quantizer with $q_{k}=\left(s_{k}-\hat{s}_{k}\right)+v_{k}$ with white noise sequence $\left\{v_{k}\right\}$, and the predictor with a strictly causal state-space system $H_{p}=\left(A_{p}, B_{p}, C_{p}, D_{p}\right)$.

decoder even though this may not be MSE minimizing.

To simplify notation greatly for this limited space, consider the encoding of a first-order AR process $s_{k}=\alpha s_{k-1}+n_{k}$ with a first-order predictor. With quantization as shown,

$$
\hat{s}_{k}=\beta \hat{s}_{k-1}+q_{k} \quad \text { where } \quad q_{k}=Q\left(s_{k}-\hat{s}_{k}\right) .
$$

Losses of $q_{k}$ s complicate the estimation process. We model the quantization error as a stochastic process with mean zero and known variance (determined by the coarseness of the quantizer $Q$ ) that is independent of $\left\{n_{k}\right\}$. Then the optimal linear estimate of $\left\{s_{k}\right\}$ is computed by a Kalman filter. Regardless of the estimation technique, the effect of a loss persists in $\hat{s}_{k+1}, \hat{s}_{k+2}, \ldots$, with a decay determined by $\beta$. It is intuitive that lowering the prediction gain $\beta$ increases the robustness to loss. Thus, the optimal $\beta$ must be a decreasing function of the loss probability $p$. However, there is no evident way to find optimal $\beta$ s without resorting to pseudorandom simulations. The computation methodology presented here allows such an overall system optimization.

Combining general state-space models for source and predictor, one can produce an overall state-space model for the generation of the $q_{k} \mathrm{~s}$. The key observation is that a Markov loss process for the channel makes the system including transmission a Markov jump linear system. The main result of [1] then shows how to bound the asymptotic average mean-square estimation error in terms of a matrix optimization problem with coupled Riccati linear matrix inequality (LMI) constraints. The optimization itself has an efficient numerical solution [2].

\section{REFERENCES}

[1] A. K. Fletcher, S. Rangan, and V. K Goyal, "Estimation from lossy sensor data: Jump linear modeling and Kalman filtering," in Inform. Process. in Sensor Netw., Berkeley, CA, Apr. 2004.

[2] S. P. Boyd, L. El Ghaoui, E. Feron, and V. Balakrishnan, Linear Matrix Inequalities in System and Control Theory, SIAM, Philadelphia, PA, 1994. 